\title{
The Impact of Covid-19 on Inmate Trust in Health Care and Willingness to Seek Treatment - A Qualitative Investigation
}

\author{
Valarie Kumalo', Alexander Nelson', Niki Messmore' \\ Indiana University School of Medicine
}

\section{Background and Objective:}

The coronavirus has disproportionately impacted vulnerable members of society. With the U.S. as the global leader in incarceration and the difficulties prison systems face implementing many of the mitigation strategies employed by the general population, the incarcerated population is in a uniquely vulnerable position particularly within an already strained prison healthcare system. Given the nature of prisons as total institutions, negative experiences with the virus coupled with a lack of autonomy could lead to a loss of trust in the healthcare system which has the potential to impact health-seeking behaviors. In this study, we aim to examine the effect of Covid-19 on Indiana inmates' trust in healthcare.

\section{Project Methods:}

Data from 380 surveys distributed across Indiana's prison system will be collected and analyzed. These results will be used to develop an interview protocol to conduct at least 25 indepth interviews which will then be coded using NVivo to identify any emerging themes regarding their experiences with Covid-19. Prior to this, in-depth literature reviews were done on health care in prison populations and trust in healthcare.

\section{Results:}

The surveys and interview protocol will be developed in the ensuing months and thus no data has yet been collected. The literature review revealed that the quality and accessibility of care in prisons is lacking, an issue exacerbated by the pandemic. Because of the disproportionate number of preexisting issues, inmates worried about the ability of prison administrators to properly protect inmates from contracting the virus. Additionally, it was found that trust likely does have an effect on health, and that many commonalities of inmates are poor predictors for trust.

\section{Potential Impact:}

This study aims to identify potential loss of trust in healthcare systems to inform community reentry programs in developing strategies that prioritize inmate health needs and perceptions. 